\title{
Case Report \\ Surgical Treatment of a Giant Liposarcoma in a Japanese Man
}

\author{
Yasuhiro Hashimoto, ${ }^{1}$ Shingo Hatakeyama, ${ }^{1}$ Tokushi Tachiwada, ${ }^{1}$ Takahiro Yoneyama, ${ }^{1}$ \\ Takuya Koie, ${ }^{1}$ Noritaka Kamimura, ${ }^{1}$ Takeshi Yanagisawa, ${ }^{1,2}$ Kenichi Hakamada, ${ }^{1,3}$ \\ and Chikara Ohyama ${ }^{1}$
}

${ }^{1}$ Department of Urology, Hirosaki University Graduate School of Medicine, 5 Zaifu-cho, Hirosaki 036-8562, Japan
${ }^{2}$ Department of Urology, Aomori Rosai Hospital, Hachinohe 031-8551, Japan
${ }^{3}$ Department of Gastroenterological Surgery, Hirosaki University Graduate School of Medicine, 5 Zaifu-cho, Hirosaki 036-8562, Japan

Correspondence should be addressed to Yasuhiro Hashimoto, bikkuri@opal.plala.or.jp

Received 19 August 2010; Revised 23 October 2010; Accepted 13 November 2010

Academic Editor: Peter E. Clark

Copyright ( 2010 Yasuhiro Hashimoto et al. This is an open access article distributed under the Creative Commons Attribution License, which permits unrestricted use, distribution, and reproduction in any medium, provided the original work is properly cited.

We report a case of a rapidly progressing giant retroperitoneal liposarcoma weighing $22 \mathrm{~kg}$ in a 41-year-old Japanese man, successfully treated with surgical excision. To our knowledge, this is the largest liposarcoma in the Japanese population reported in the literature.

\section{Introduction}

Liposarcoma is a common soft tissue sarcoma which occurs in adults $[1,2]$. Its common sites of occurrence are the extremities, retroperitoneum, and inguinal region [3]. Although liposarcomas can often grow into large tumors, resected tumors weighing over $20 \mathrm{~kg}$ are extremely rare and considered to be "giant liposarcomas." Here, we report our experience of a giant retroperitoneal liposarcoma, presumably the largest reported from Japan; it weighed $22 \mathrm{~kg}$ and caused abdominal swelling, marked leg edema, and cough due to the pressure it exerted on the patient's diaphragm.

\section{Case Presentation}

A 41-year-old Japanese man became aware of abdominal swelling in December 2008 but did not seek a medical opinion until he developed marked leg edema, a $30 \mathrm{~kg}$ weight gain, and cough. He was hospitalized the same day after a retroperitoneal tumor was detected by computed tomography (CT). After hospitalization, the patient was diagnosed with a liposarcoma by CT-guided needle biopsy. The patient was emergently transferred to our hospital due to worsening respiratory status. On admission his body surface area was $2.18 \mathrm{~m}^{2}$. A CT scan detected a $43 \times 37 \times 31 \mathrm{~cm}$ tumor in the right retroperitoneum, with the right kidney significantly displaced to the left and anterior side (Figure 1). Whole-body CT scan detected no metastasis. His condition was diagnosed as respiratory failure due to elevation of the diaphragm as a consequence of the rapid growth of the tumor (Figure 2).

We concluded that surgical resection was the only option for saving the patient's life, so we performed a tumorectomy 3 weeks later through a midline incision. The operation took 3 hours and 20 minutes, and the estimated blood loss was $3990 \mathrm{~mL}$ requiring $800 \mathrm{~mL}$ blood transfusion. During surgery, the tumor and right kidney were completely resected.

Pathological examination showed that the resected mass weighed $22 \mathrm{~kg}$ and was $45 \times 40 \times 30 \mathrm{~cm}$ in size (Figure 3). Grossly, the tumor was well encapsulated and transverse sectioning revealed a solid multinodular tumor mass. Histopathologic analysis demonstrated many spindle cells with high-grade atypia; some of which were lipoblasts on a myxoid background; there were no split signals on FISH analysis using CHOP DNA probes, thus leading to the diagnosis of dedifferentiated liposarcoma (Figure 4). Microscopically, no tumor cells were identified in the right kidney and this was an R0 resection. He declined adjuvant therapy. The patient was discharged from our hospital after 


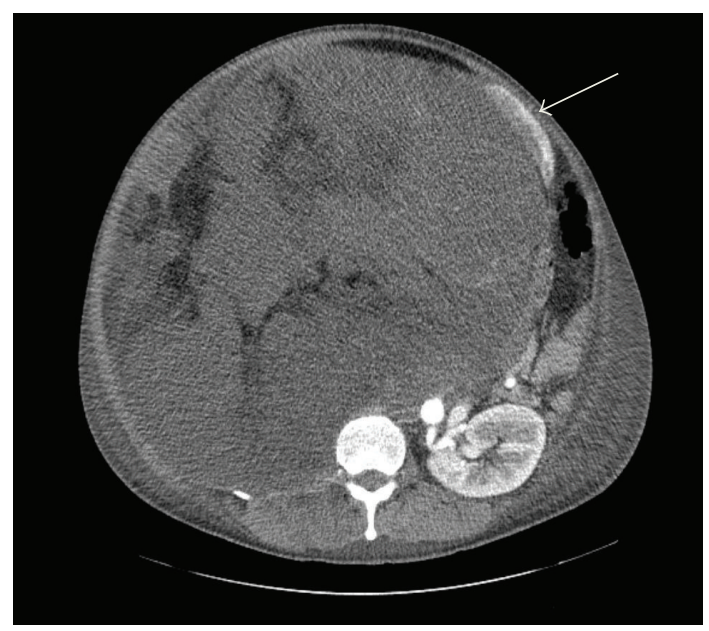

Figure 1: Computerized tomography detected a $43 \times 37 \times 31$ $\mathrm{cm}$ tumor in the right retroperitoneum, and the right kidney was deflected greatly to the left ventral side (white arrow).

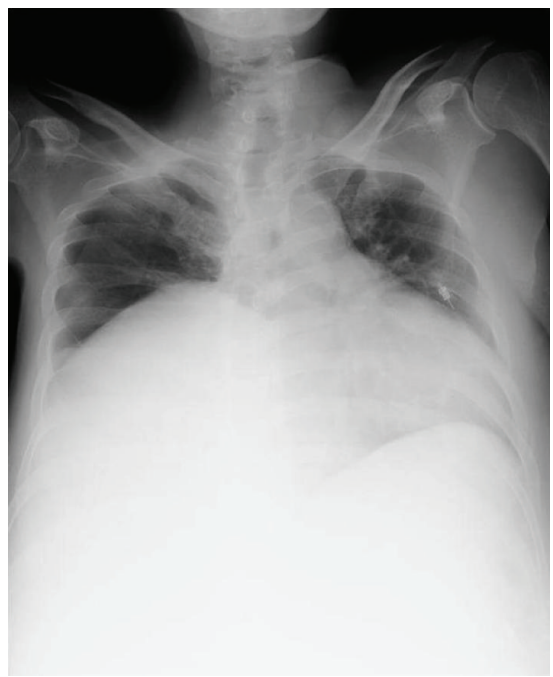

Figure 2: Chest X-ray showed that elevation of the diaphragm due to rapid growth of retroperitoneal tumor and cardiac enlargement.

18 days. His body surface area at discharge was $1.66 \mathrm{~m}^{2}$. A CT scan performed 12 months after surgery detected no recurrence.

\section{Discussion}

Liposarcoma is the most common soft tissue sarcoma and accounts for $10-20 \%$ of all cases. It commonly occurs between 40-60 years of age and has a 1:1 ratio between genders $[1,2]$. Liposarcoma occurs most commonly in the extremities (52\%), retroperitoneum (19\%), and inguinal region (12\%) [3]. Although liposarcomas sometimes weigh as much as $10 \mathrm{~kg}$ or more, those exceeding $20 \mathrm{~kg}$ are extremely rare. Some published reports have noted tumors weighing $103.6 \mathrm{lb}(46.6 \mathrm{~kg}$ ) [4], $42 \mathrm{~kg}$ [5], and $18 \mathrm{~kg}$ [6]. According to previous reports, the heaviest tumor to date in

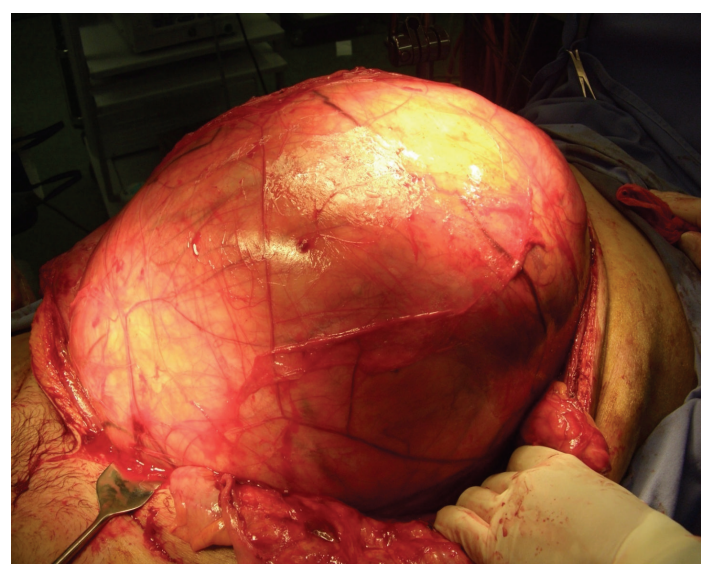

FIgure 3: Intra-operative findings. The retroperitoneal tumor weighing $22 \mathrm{~kg}$ was extirpated.

the Japanese population was $18 \mathrm{~kg}$ [7]. Thus, we believe that we have resected the largest retroperitoneal liposarcoma in Japan.

According to the WHO classification, liposarcomas can be classified into five histologic types: well differentiated, myxoid, round cell, pleomorphic, and dedifferentiated [8]. The myxoid and round-cell types are considered subtypes because of their common chromosomal translocation [912].

Dalal argued, on the basis of a study conducted at a single facility, that the most common forms of liposarcoma are well differentiated (46\%), dedifferentiated (18\%), myxoid (18\%), round cell $(10 \%)$, and pleomorphic $(8 \%)$.

A chimera gene, TLS-CHOP, which is only found in myxoid-type sarcomas, was discovered in a myxoid-type liposarcoma. This gene is presumed to be responsible for tumor growth [9-12]. It can often be difficult to distinguish between myxoid-type and dedifferentiated liposarcomas. Nevertheless, we observed no split signals on FISH analysis using CHOP DNA probes.

Surgical resection is still the principal treatment, and combined resection of involved organs is necessary for an $\mathrm{R} 0$ resection in many cases. A previous report stated that combined resection of adjacent organs is required in $75 \%$ of retroperitoneal tumors treated by tumorectomy [13].

Multimodality therapies such as chemotherapy with ifosfamide, anthracycline, and doxorubicin, as well as the combination of intraoperative and intensity-modulated radiotherapy, are also effective [14]. Recently, Ecteinascidin (ET743), an alkaloid derived from the Caribbean sea squirt (Ecteinascidia turbinate), has shown definite activity in soft tissue sarcoma in clinical trials [14]. However, high-grade liposarcoma may benefit from radiotherapy or chemotherapy with a reduction in local recurrence, but with no benefit in overall survival [14]. Histologic grading is important in liposarcoma, with disease-specific survival rates after 5 years of $93 \%$ for well-differentiated, $74-92 \%$ for myxoid/roundcell, 59\% for pleomorphic, and 44\% for dedifferentiated forms [15]. $83 \%$ of patients with dedifferentiated liposarcomas develop local recurrence, and 30\% develop distant 


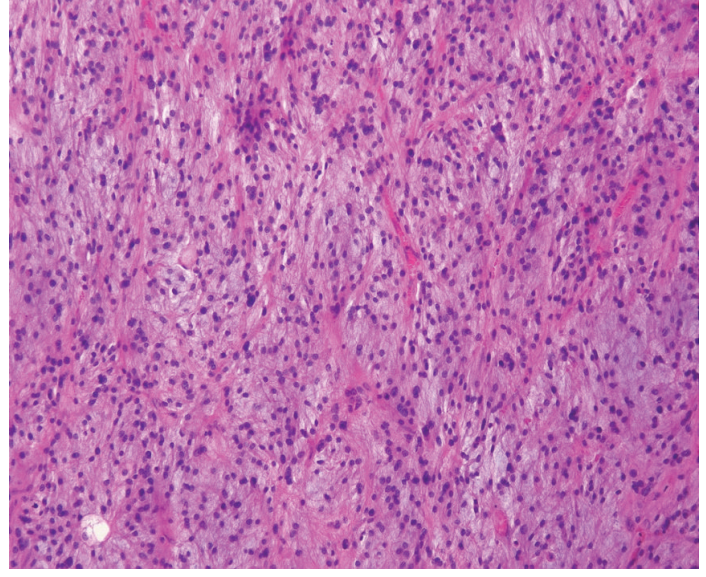

(a)

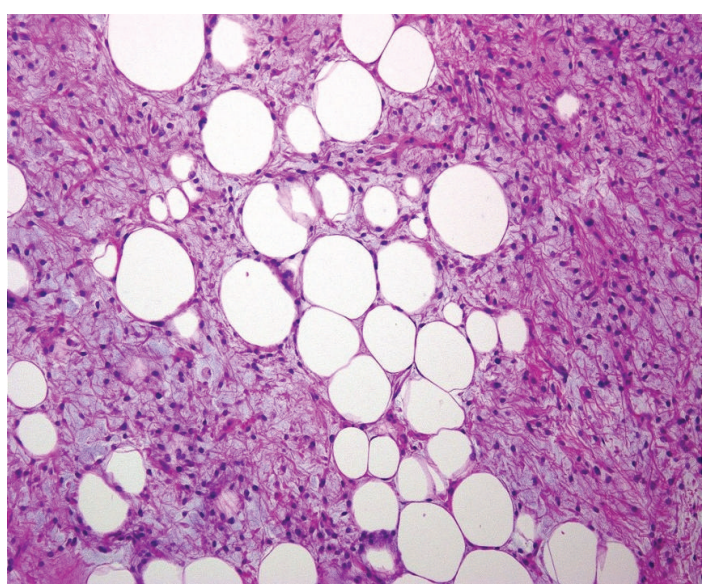

(b)

Figure 4: Histopathological findings of the tumor diagnosed dedifferentiated liposarcoma (HE stain, original magnification x200). (a) Most of the tumor cells consisted of spindle cells with highgrade atypia. (b) Some of which were identified to be lipoblasts in a myxoid background.

metastasis [16]. Here, we report the largest retroperitoneal liposarcoma in Japan. Judging from evidence in previously published reports, we expect a poor prognosis for the patient and will be closely following him for recurrence.

\section{References}

[1] T. M. Mack, "Sarcomas and other malignancies of soft tissue, retroperitoneum, peritoneum, pleura, heart, mediastinum, and spleen," Cancer, vol. 75, no. 1, pp. 211-244, 1995.

[2] W. M. Mendenhall, R. A. Zlotecki, S. N. Hochwald, A. W. Hemming, S. R. Grobmyer, and W. G. Cance, "Retroperitoneal soft tissue sarcoma," Cancer, vol. 104, no. 4, pp. 669-675, 2005.

[3] W. O. Russell, J. Cohen, F. Enzinger et al., "A clinical and pathological staging system for soft tissue sarcomas," Cancer, vol. 40, no. 4, pp. 1562-1570, 1977.

[4] O. J. McCallum, J. J. Burke 2nd, A. J. Childs, A. Ferro, and D. G. Gallup, "Retroperitoneal liposarcoma weighing over one hundred pounds with review of the literature," Gynecologic Oncology, vol. 103, no. 3, pp. 1152-1154, 2006.
[5] S. Yol, S. Tavli, L. Tavli et al., "Retroperitoneal and scrotal giant liposarcoma: report of a case," Surgery Today, vol. 28, pp. 339342, 1998.

[6] A. Herrera-Gomez, C. Ortega-Gutierrez, A. M. Betancourt, and K. Luna-Ortiz, "Giant retroperitoneal liposarcoma," World Journal of Surgical Oncology, vol. 6, article 115, 2008.

[7] K. Inoue, Y. Higaki, and H. Yoshida, "Giant retroperitoneal liposarcoma," International Journal of Urology, vol. 12, no. 2, pp. 220-222, 2005.

[8] C. Fletcher, Pathology and Genetics of Tumors of Soft Tissue and Bone., International Agency for Research on Cancer Press, Lyon, France, 2002.

[9] S. Heim and F. Mitelman, Cancer Cytogenetics, Wiley-Liss, New York, NY, USA, 2nd edition, 1995.

[10] P. Aman, D. Ron, N. Mandahl et al., "Rearrangement of the transcription factor gene CHOP in myxoid liposarcomas with $\mathrm{t}(12 ; 16)(\mathrm{q} 13 ; \mathrm{p} 11)$," Genes Chromosomes and Cancer, vol. 5, no. 4, pp. 278-285, 1992.

[11] A. Crozat, P. Aman, N. Mandahl, and D. Ron, "Fusion of CHOP to a novel RNA-binding protein in human myxoid liposarcoma," Nature, vol. 363, no. 6430, pp. 640-644, 1993.

[12] T. H. Rabbitts, A. Forster, R. Larson, and P. Nathan, "Fusion of the dominant negative transcription regulator $\mathrm{CHOP}$ with a novel gene FUS by translocation $\mathrm{t}(12 ; 16)$ in malignant liposarcoma," Nature Genetics, vol. 4, no. 2, pp. 175-180, 1993.

[13] J. W. Kilkenny 3rd, K. I. Bland, and E. M. Copeland 3rd, "Retroperitoneal sarcoma: the University of Florida experience," Journal of the American College of Surgeons, vol. 182, no. 4, pp. 329-339, 1996.

[14] K. M. Skubitz and D. R. D’Adamo, "Sarcoma," Mayo Clinic Proceedings, vol. 82, no. 11, pp. 1409-1432, 2007.

[15] K. M. Dalal, M. W. Kattan, C. R. Antonescu, M. F. Brennan, and S. Singer, "Subtype specific prognostic nomogram for patients with primary liposarcoma of the retroperitoneum, extremity, or trunk," Annals of Surgery, vol. 244, no. 3, pp. 381389, 2006.

[16] K. M. Dalal, C. R. Antonescu, and S. Singer, "Diagnosis and management of lipomatous tumors," Journal of Surgical Oncology, vol. 97, no. 4, pp. 298-313, 2008. 


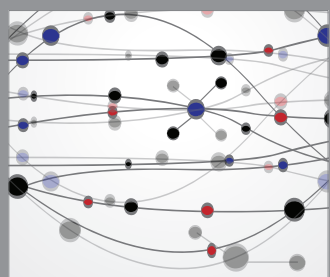

The Scientific World Journal
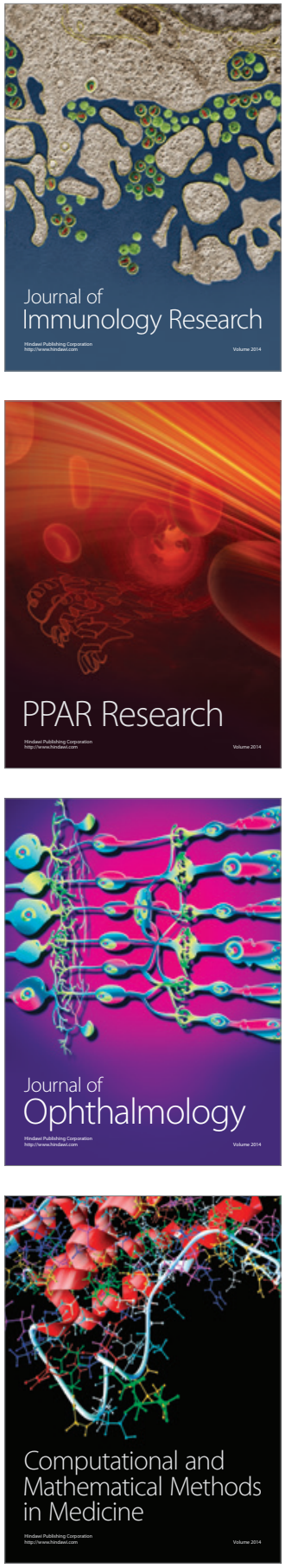

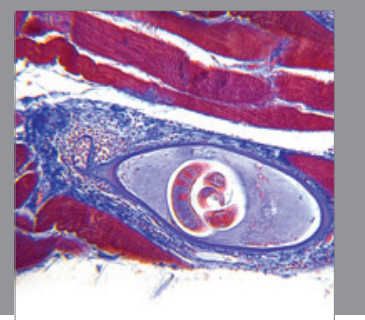

Gastroenterology

Research and Practice
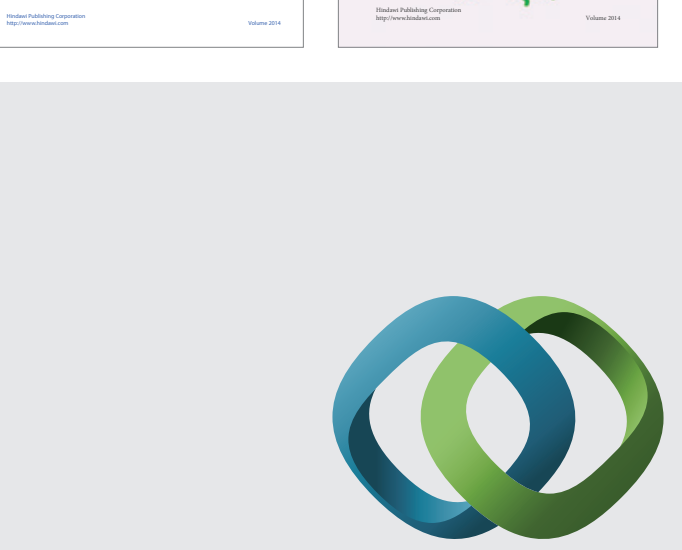

\section{Hindawi}

Submit your manuscripts at

http://www.hindawi.com
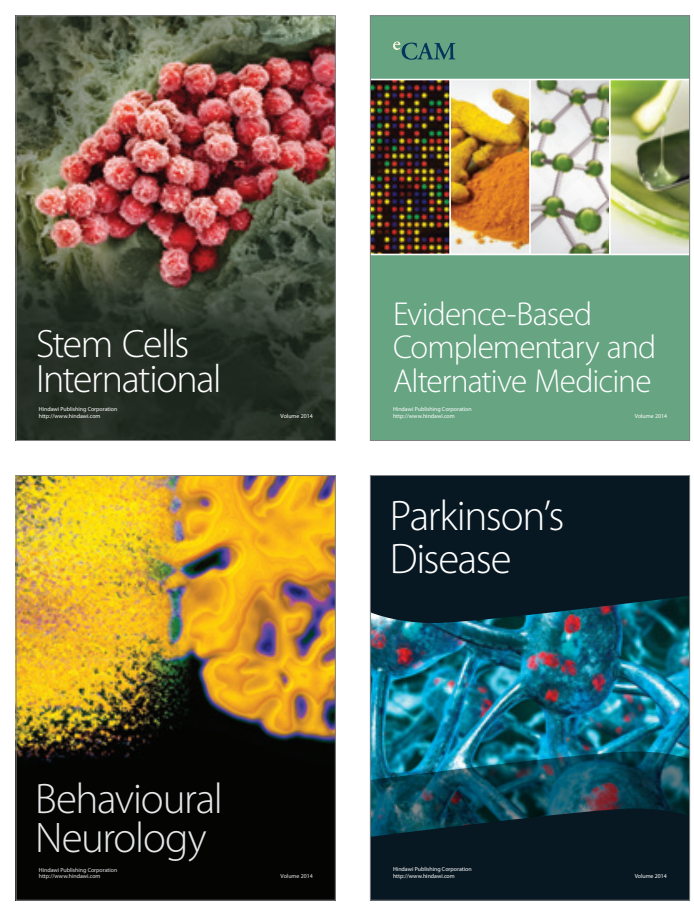

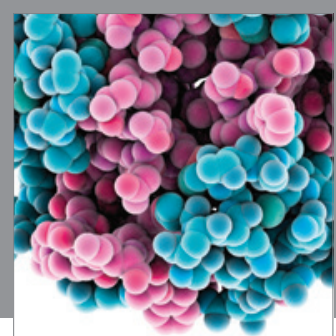

Journal of
Diabetes Research

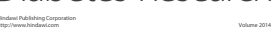

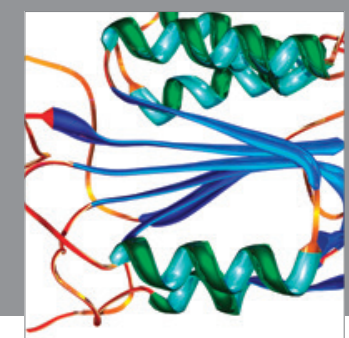

Disease Markers
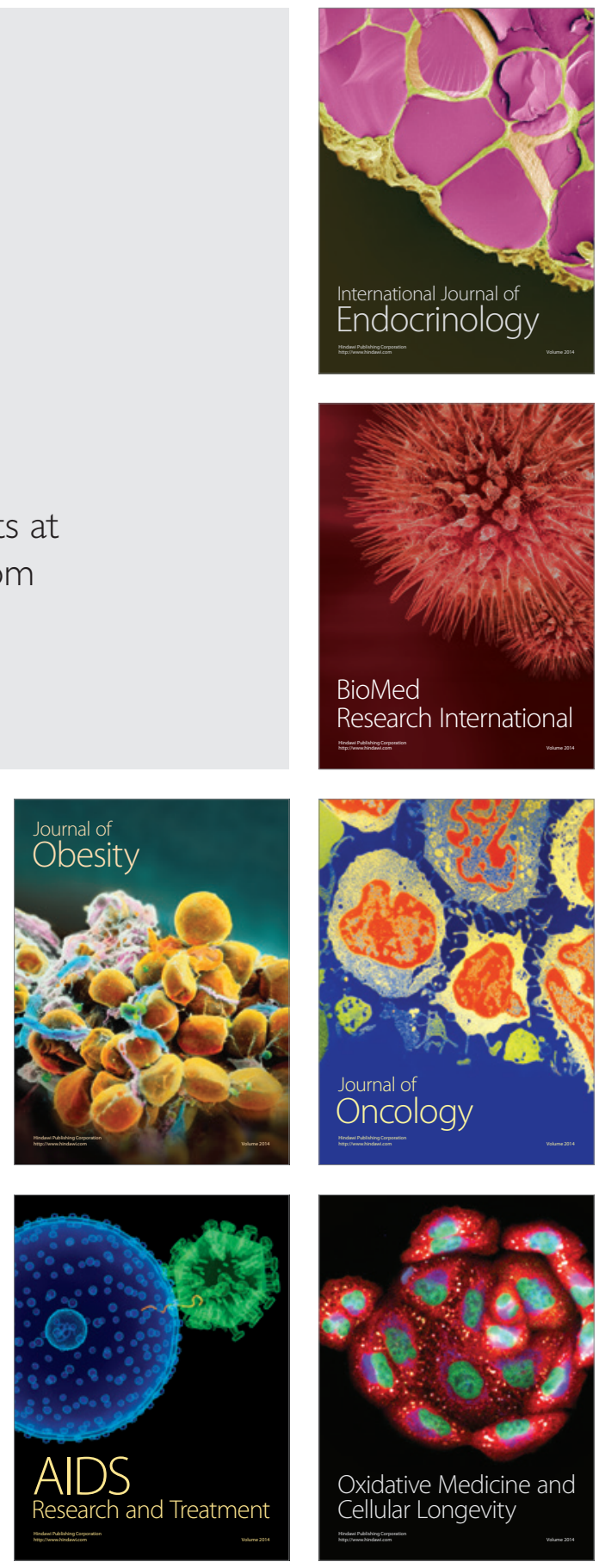